\title{
Initial LDH level can predict the survival benefit from bevacizumab in the first-line setting in Chinese patients with metastatic colorectal cancer
}

\author{
This article was published in the following Dove Press journal: \\ OncoTargets and Therapy \\ II August 2014 \\ Number of times this article has been viewed
}

\section{Chenxi Yin ${ }^{1,2, *}$ \\ Chang Jiang ${ }^{1,2, *}$ \\ Fangxin Liao ${ }^{1,2}$ \\ Yuming Rong ${ }^{1,2}$ \\ Xiuyu Cai ${ }^{1,2}$ \\ Guifang Guo, 1,2 \\ Huijuan Qiu',2 \\ Xuxian Chen ${ }^{1,2}$ \\ Bei Zhang ${ }^{1,2}$ \\ Wenzhuo $\mathrm{He}^{1,2}$ \\ Liangping $\mathrm{Xia}^{1,2}$}

'State Key Laboratory of Oncology in South China, Sun Yat-sen University

Cancer Center, Collaborative

Innovation Center for Cancer,

Medicine, ${ }^{2}$ VIP Region, Sun Yat-sen

University Cancer Center, Guangzhou,

Guangdong, People's Republic of

China

*These authors contributed equally to this paper

\begin{abstract}
Background: Markers to predict the efficacy of bevacizumab treatment have been not fully validated in most cancers, including metastatic colorectal cancer (mCRC). The aim of this study was to investigate the potential role of lactate dehydrogenase (LDH) in predicting the survival benefit from first-line bevacizumab treatment, in Chinese patients with $\mathrm{mCRC}$.

Methods: All the patients were diagnosed with mCRC at the Sun Yat-sen University Cancer Center from 2003 to 2013. The study group and the control group were classified by receiving bevacizumab or not. The serum LDH value of all the patients had been detected before the first-line treatment. The primary end point was progression-free survival (PFS).

Results: The median PFS of the study and the control group (patients who received bevacizumab or not) was 11.3 and 9.1 months, respectively $(P=0.004)$. In the control group, the median PFS of the high LDH level and the low LDH level groups was 6.9 and 10.2 months, respectively $(P<0.001)$. However, in the study group, the corresponding median PFS was 9.9 and 11.9 months, respectively $(P=0.145)$. In addition, for the low LDH level group, the median PFS was 11.9 and 10.2 months for patients who received bevacizumab or not, respectively $(P=0.066)$; however, the median PFS of patients receiving bevacizumab or not was significantly different in the high LDH level group (9.9 and 6.9 months, respectively) $(P=0.012)$.

Conclusion: The addition of bevacizumab in the first-line treatment setting could improve the PFS of mCRC patients notably. However, the benefit could only be potentially reflected on patients with high serum LDH level.
\end{abstract}

Keywords: lactate dehydrogenase, PFS, progression-free survival

\section{Introduction}

Bevacizumab, the classical antiangiogenesis drug, is widely used in first-line treatment, ${ }^{1,2}$ in second-line treatment, ${ }^{3}$ and even after progression beyond its failure, in metastatic colorectal cancer (mCRC). ${ }^{4}$ However, implementing bevacizumab treatment has always been challenged. Though both progression-free survival (PFS) and overall (OS) were improved significantly in the AVF2017, ARTIST, and E3200 trials, ${ }^{1,3,5}$ the original short OS of the irinotecan/leucovorin/fluorouracil (IFL) regimen in the former two trials weakened their significance. Further, only the PFS was statistically significant, while the OS was not in the NO16966 trial. For similar reasons, the US Food and Drug Administration (FDA) withdrew the license for bevacizumab in metastatic breast cancer when combined with paclitaxel in the first-line treatment setting. ${ }^{6-9}$ The reason may be that there is not yet an appropriate marker that will select patients who will 
benefit from bevacizumab, in spite of the abundant studies carried out in past years.

Hypoxia, aggravated by bevacizumab ${ }^{10}$ because of pruning immature vessels, contributes to the resistance of cancer to chemotherapy ${ }^{11}$ and radiotherapy. ${ }^{12}$ In order to adapt the anoxic microenvironment and compete with the surrounding normal cells for resources for survival, cancer cells choose aerobic glycolysis to guarantee the high speed of proliferation. ${ }^{13} \mathrm{LDH}$ (lactate dehydrogenase), the main catalyst of aerobic glycolysis, has been demonstrated to be a poor prognostic factor of many cancers, ${ }^{14-21}$ and a high level of serum LDH has been associated with poorer survival. Moreover, the relationship between LDH and bevacizumab has been explored. The hypoxia-inducible factor (HIF) gene was found to be stimulated by aerobic glycolysis, which upregulated hundreds of downstream genes, including those for LDH and vascular endothelial growth factor (VEGF). ${ }^{22}$ Meanwhile, high serum LDH level was also confirmed to be associated with the overexpression of VEGFA and VEGFR-1 in tumor. ${ }^{23}$ Considering the correlation of LDH and VEGF, several studies have tried to investigate the predictive value in the antiangiogenic therapy, including bevacizumab. Both Hecht et $\mathrm{al}^{24}$ and Van Cutsem et $\mathrm{al}^{25}$ investigated the median PFS of placebo or vatalanib - an oral inhibitor of VEGF receptors - in combination with leucovorin/fluorouracil/ oxaliplatin regimen in first- and second-line treatment of advanced colorectal cancer. The initial end point of OS was not met in the two trials; however, in an exploratory post hoc analysis, the PFS was found to be improved with the use of vatalanib in patients with high LDH serum levels, which showed the potential value of LDH to predict the efficacy of vatalanib. Since bevacizumab targets VEGF as well, the question is whether LDH has the same power to predict its efficacy in mCRC. To the best of our knowledge, only one study has focused on this topic so far. Scartozzi et $\mathrm{al}^{23}$ found that the PFS in the high LDH level group (cutoff point 588 $\mathrm{mg} / \mathrm{dL}$ ) was much shorter than in the low LDH level group, in the patients not receiving bevacizumab, while in the patients receiving bevacizumab, the PFS could not be distinguished by the two different LDH levels. However, the main point of who can get benefit from bevacizumab, among patients with high or low LDH level, has not been investigated yet. Therefore, we conducted the current study.

\section{Patients and methods}

\section{Study population}

Patients who met the following criteria were selected as the study group: 1) diagnosed with mCRC, based on pathological specimens from the primary tumor and clinical and/or pathological evidence of distant metastasis, at the Sun Yat-sen University Cancer Center from 2003 to 2013; 2) have finished an entire course of first-line chemotherapy in the center; 3) Eastern Cooperative Oncology Group (ECOG) performance status $\leq 2$; 4) bevacizumab was used as a combination with the standard first-line regimens including oxaliplatin-based regimens and irinotecanbased regimens. More than four cycles of bevacizumab were required, since four cycles of bevacizumab was the minimum requirement to benefit patients found in our previous study; ${ }^{26} 5$ ) serum LDH was tested before the initiation of the first-line chemotherapy. The consecutive mCRC patients in the control group met the same criteria as the study group except for bevacizumab administration. The exclusion criteria of both groups included 1) lack of a pathological diagnosis; 2) incomplete medical history; 3) loss to follow-up; 4) suffering from two or more kinds of tumors asynchronously or synchronously.

\section{Laboratory measurement of serum LDH}

LDH was included in the serum biochemical tests in our cancer center. The serum biochemical tests were performed using a Hitachi Clinical Analyzer 7600-020 (Hitachi High-Technologies Corp, Tokyo, Japan). The normal range is $109-245 \mathrm{U} / \mathrm{L}$. LDH levels in the current study were classified into two groups, the high LDH level group was above the upper limit of normal value, while the low LDH level group was within and below the normal value.

\section{Follow-up and statistical analyses}

The last follow-up assessment was conducted on October 31, 2013, through telephone interview or medical records review. All the patients of the study and the control group had progressed by the time of follow-up. The end point of the study was PFS, deemed to be the length of time from the initial treatment of the first-line therapy to the date of progression by imaging examination, according to the Response Evaluation Criteria in Solid Tumors (RECIST), ${ }^{27}$ or to the death of the patient. The weight of each of the factors was tested by the chi-square test, and the PFS was compared using the Kaplan-Meier method log-rank test, which included calculations of medians and survival curves. All the statistical analysis was conducted using the SPSS for Windows, Version 18.0 software package (IBM, Armonk, NY, USA), and a $P$-value less than 0.05 was considered with statistically significant. 


\section{Results}

\section{Patient characteristics}

There were 87 and 246 patients included in the study group (receiving bevacizumab) and the control group (not receiving bevacizumab), respectively. The patient characteristics and potential prognostic factors, including sex, age, tumor location, pathological type, and chemotherapy regimens are shown in Table 1. All of these factors were balanced in statistics, except for pathological type and chemotherapy regimen. However, these latter two factors were statistically insignificant with respect to PFS ( $P$-values were 0.093 and 0.482 , respectively).

\section{PFS of patients treated with and without bevacizumab}

In the study group, bevacizumab was combined with oxaliplatin-based, irinotecan-based, or fluorouracil singleagent regimens in 42 (48.3\%), 43 (49.4\%), and two (2.3\%) patients, respectively (Table 1); in the control group, there were $202(82.1 \%), 37(15 \%)$, and six $(2.4 \%)$ patients treated with the three regimens, respectively; only one $(0.4 \%)$ patient was treated with cyclophosphamide and cisplatin (CTX + DDP). The median (range) PFS of the study group and the control group were 11.3 (9.9-12.6 months) and 9.1 months (7.9-10.2 months), respectively ( $P=0.004)$ (Figure 1).

Table I Patient baseline characteristics in the study group and the control group

\begin{tabular}{|c|c|c|c|c|}
\hline \multirow[t]{2}{*}{ Patient characteristics } & \multirow{2}{*}{$\begin{array}{l}\text { Study } \\
\text { group (\%) }\end{array}$} & \multirow{2}{*}{$\begin{array}{l}\text { Control } \\
\text { group (\%) }\end{array}$} & \multicolumn{2}{|c|}{$P$-value* } \\
\hline & & & $T$ & 2 \\
\hline Number & $87(26.1)$ & 246 (73.9) & & \\
\hline Sex & & & 0.972 & 0.960 \\
\hline Male & $55(63.2)$ & $155(63)$ & & \\
\hline Female & $32(36.8)$ & 91 (37) & & \\
\hline \multicolumn{5}{|l|}{ Age (years) } \\
\hline Median (range) & $52(19-83)$ & $51(25-77)$ & 0.998 & 0.718 \\
\hline$\leq 60$ & $65(73.9)$ & I8I (73.6) & & \\
\hline$>60$ & $23(26.1)$ & $65(26.4)$ & & \\
\hline Primary tumor & & & 0.299 & 0.661 \\
\hline Colon & $53(60.9)$ & $165(67.1)$ & & \\
\hline Rectum & $34(39.1)$ & 81 (32.9) & & \\
\hline \multicolumn{5}{|l|}{ Pathological type } \\
\hline Adenocarcinoma & 82 & & & \\
\hline Others & 5 & & & \\
\hline Chemotherapy regimen & & & $<0.001$ & 0.482 \\
\hline Oxaliplatin-based & $42(48.3)$ & $202(82.1)$ & & \\
\hline Irinotecan-based & $43(49.4)$ & $37(I 5.0)$ & & \\
\hline Fluorouracil alone & $2(2.3)$ & $6(2.4)$ & & \\
\hline Other type & & $\mathrm{I}(0.5)$ & & \\
\hline
\end{tabular}

Note: *I refers to the $P$-value of the balance test and 2 refers to the $P$-value of the elements to PFS.

Abbreviation: PFS, progression-free survival.

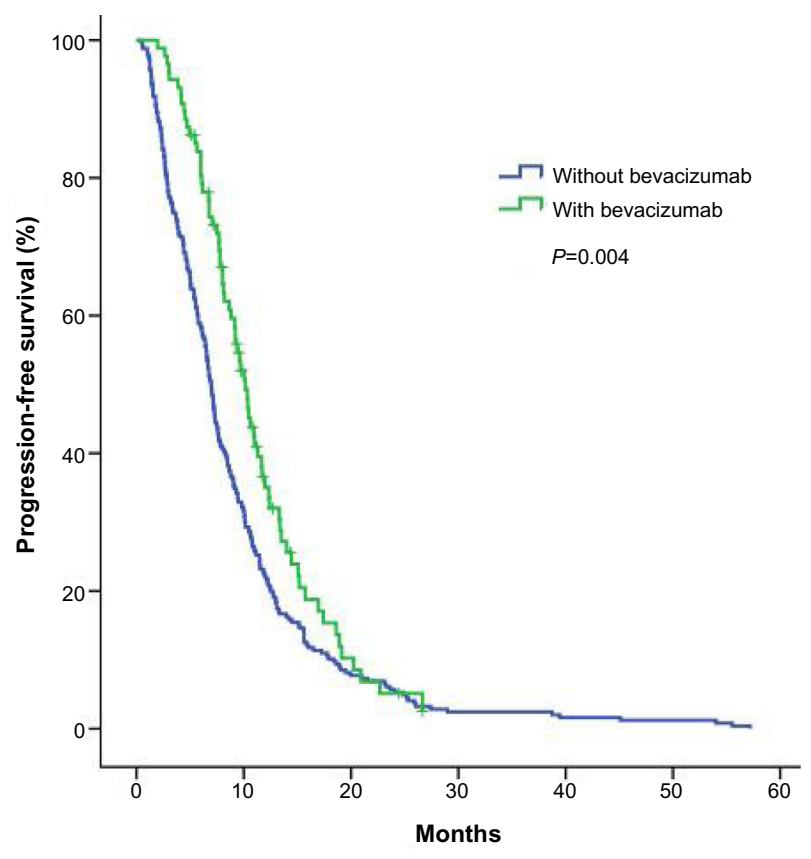

Figure I The PFS of patients with and without bevacizumab. Abbreviation: PFS, progression-free survival.

\section{PFS of patients with high and low LDH level}

It has been reported that serum LDH level was a prognostic factor in many cancers. ${ }^{28-30}$ So, we put all the patients in the study group and the control group together to investigate the role of LDH. There were 217 (65.2\%) and 116 (34.8\%) patients classified into the low LDH level group and high LDH level group, respectively. The median PFS of the patients in the two groups was 10.8 and 7.8 months, respectively $(P<0.001)$ (Figure 2$)$. Meanwhile, It means that the number of patients receiving bevacizumab or not is statistically balanced in the two LDH level groups $(P=0.936)$. In the control group, there were $86(35.0 \%)$ and $160(65.0 \%)$ patients included in the high LDH level and low level groups respectively, and their corresponding median PFS was 6.9 and 10.2 months $(P<0.001)$, respectively. In the study group, 30 (34.5\%) and 57 (65.5\%) patients were divided into the high LDH level and low level groups, and the median PFS was 9.9 and 11.9 months, respectively $(P=0.145)$ (Figure 3 ).

In the low LDH level group, receiving bevacizumab was not statistically significantly associated with PFS.

There were 217 patients in the low LDH level group, $57(26.3 \%)$, and $160(73.7 \%)$ patients of whom were in the bevacizumab or control group (no bevacizumab), respectively. The median PFS of the patients who received bevacizumab or not was 11.9 months and 10.2 months, respectively ( $P=0.066)$ (Figure 4$)$. 


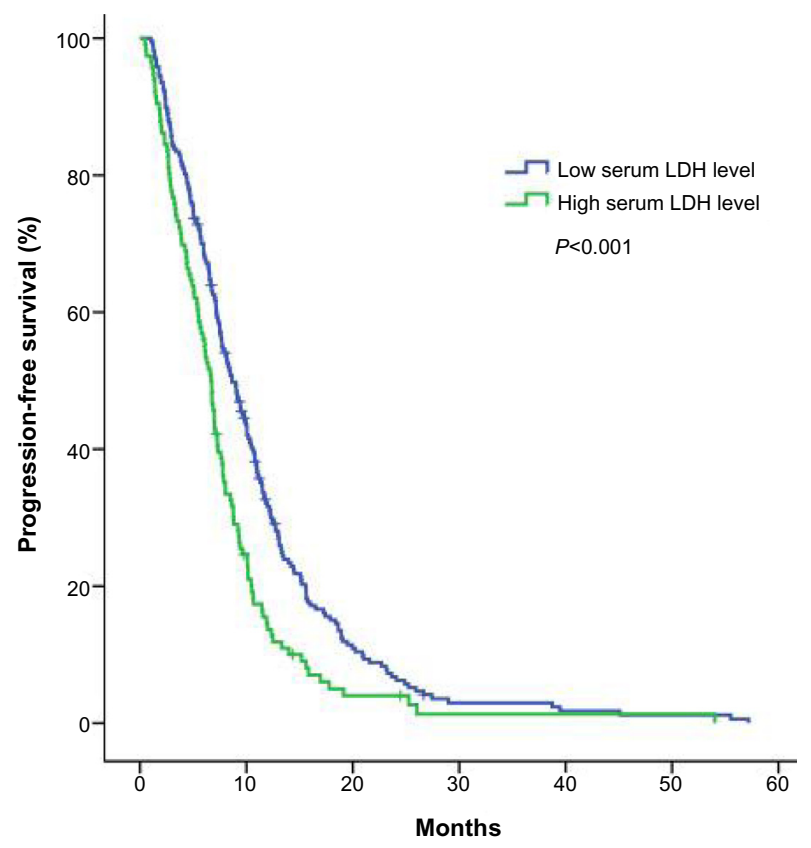

Figure 2 The PFS of patients in the high and low serum LDH level groups. Abbreviations: LDH, lactate dehydrogenase; PFS, progression-free survival.

In the high LDH level group, the receipt of bevacizumab or not had a significant impact on PFS.

There were 116 patients in the LDH high level group; the number of patients receiving bevacizumab or not was $30(25.9 \%)$ and $86(74.1 \%)$, respectively. And the median PFS was 9.9 months and 6.9 months, respectively $(P=0.012)$ (Figure 5).

Though receiving bevacizumab was not statistically significantly in the low LDH level group, some people might question the results because of the $P$-value is 0.66 , which is not particularly big. Therefore, in order to consolidate the conclusion, the whole patient sample was divided equally into three groups (low, middle, and high) according to their level of $\mathrm{LDH}$. The patient characteristics and potential prognostic factors of the three groups, including sex, age, tumor location, pathological type, and chemotherapy regimen are shown in Table 2, and all of the factors were balanced in statistics. Consistent with the other results, the gradually increasing difference of PFS in the subgroups (receipt of bevacizumab or not) was accompanied by the increasing of serum LDH in the three groups. The $P$-value of receipt of bevacizumab or not was $0.285,0.081$, and 0.006 in the serum low LDH level, middle level, and high level groups, respectively (Figure 6).

The four subgroups, including the patients of the high LDH level and the low level groups who received bevacizumab or not, respectively, were pairwise compared. It was shown that the three survival curves of patients who received bevacizumab or not in the low LDH level group and the patients who received bevacizumab in the high LDH level group were insignificant $(P=0.171)$, while the each of them is statistically significant with the group of not received bevacizumab in the high LDH level $(P<0.001)$, as shown in Figure 7.

\section{Discussion}

There have been few studies focused on the effect of bevaci-

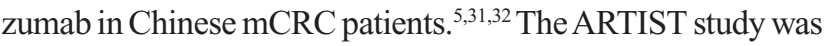
the only randomized Phase III clinical trial, and it found that
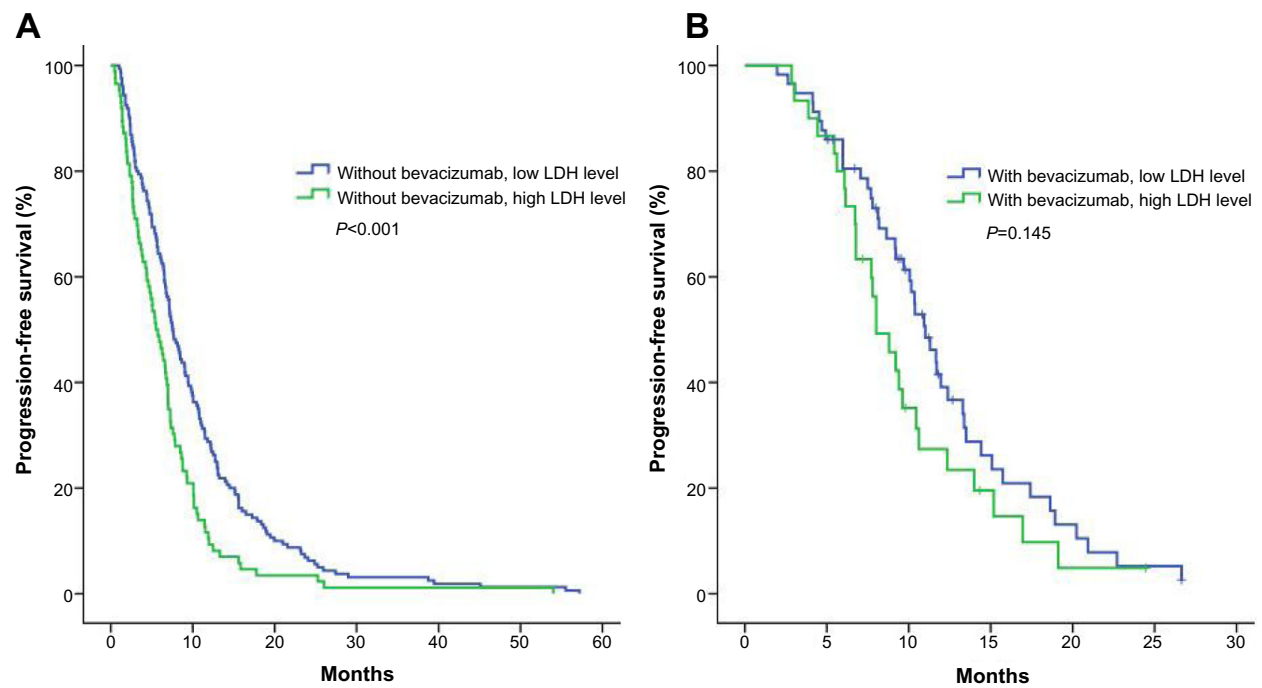

Figure 3 (A) The PFS of patients without bevacizumab, in the high and low serum LDH level groups; (B) the PFS of patients with bevacizumab, in the high and low serum LDH level groups.

Abbreviations: LDH, lactate dehydrogenase; PFS, progression-free survival. 


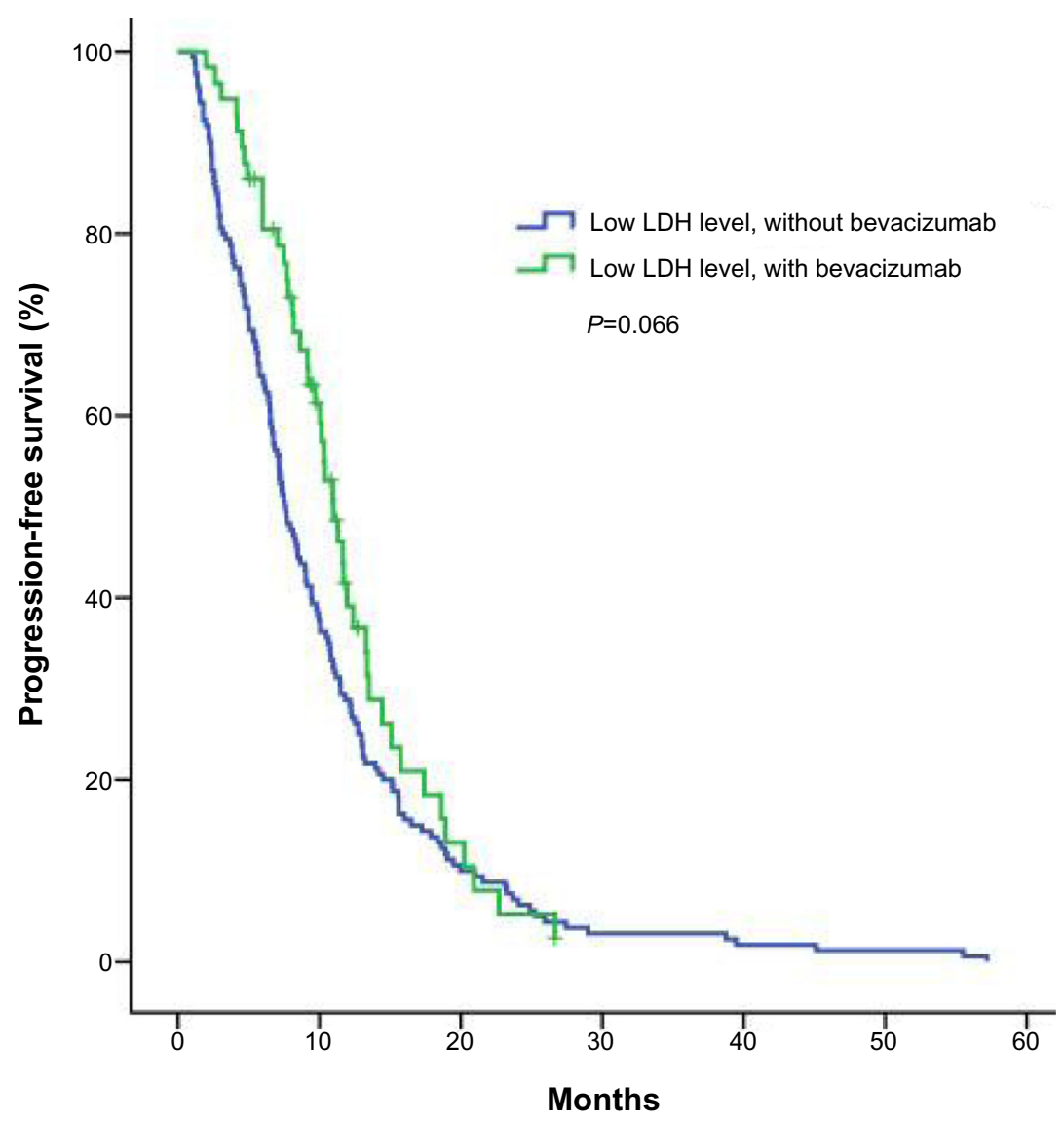

Figure 4 The PFS of patients with or without bevacizumab, in the low serum LDH level group. Abbreviations: LDH, lactate dehydrogenase; PFS, progression-free survival.

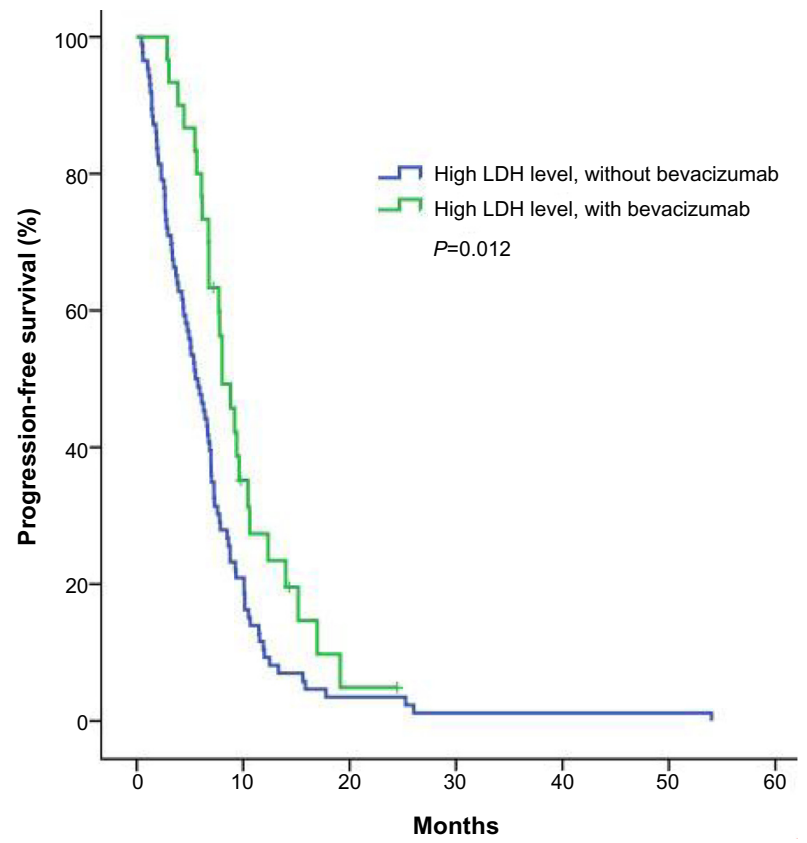

Figure 5 The PFS of patients with or without bevacizumab, in the high serum LDH level group.

Abbreviations: LDH, lactate dehydrogenase; PFS, progression-free survival. both OS and PFS were improved with the addition of bevacizumab. ${ }^{5}$ Then, our previous case-control study, which analyzed data from patients before 2010, found that when bevacizumab was used for at least four cycles, there was an improvement of OS, although the PFS was not investigated. ${ }^{31}$ Recently, a retrospective study with a small patient number showed the efficacy and safety profiles of bevacizumab in first-line, second-line, and even the third-line treatment in Chinese patients. ${ }^{32}$ Usually, OS and/or PFS have been set as the end points to evaluate the efficacy of drugs. However, only 23 (26.4\%) patients had died (bevacizumab group) at the end of follow-up in our current study. As a result, PFS was chosen as the end point. In accordance with the studies above, the current study also demonstrated the efficacy of bevacizumab, though the distributions of pathological type and chemotherapy regimens were not balanced in statistics. However, neither of these had a statistically significant impact on PFS. A predictive marker of the efficacy of bevacizumab has not been established and firmly validated on account of the complex tumor environment ${ }^{33}$ and network of tumor angiogensis. ${ }^{34}$ Bevacizumab may be able to, not only 
Table 2 Patient baseline characteristics in the serum LDH low, middle, and high levels

\begin{tabular}{|c|c|c|c|c|c|}
\hline \multirow[t]{2}{*}{ Patient characteristics } & \multirow[t]{2}{*}{ All patients } & \multicolumn{3}{|c|}{ Serum LDH level } & \multirow[t]{2}{*}{$P$-value } \\
\hline & & Low (\%) & Middle (\%) & High (\%) & \\
\hline Number & 333 & III (33.3) & III (33.3) & III (33.3) & \\
\hline Sex & & & & & 0.110 \\
\hline Male & 210 & $77(69.4)$ & $71(64.0)$ & $62(55.9)$ & \\
\hline Female & 123 & $34(30.6)$ & $40(36.0)$ & $49(44.1)$ & \\
\hline Age (years) & & & & & 0.074 \\
\hline Median (range) & $52(19-83)$ & $49(19-83)$ & $54(29-78)$ & $53(|9-8|)$ & \\
\hline$\leq 60$ & 245 & $89(80.2)$ & $74(66.7)$ & $82(73.9)$ & \\
\hline$>60$ & 88 & $22(19.8)$ & $37(33.3)$ & $29(26.1)$ & \\
\hline Primary tumor & & & & & $0.41 \mathrm{I}$ \\
\hline Colon & 218 & $78(70.3)$ & $71(64.0)$ & $69(62.2)$ & \\
\hline Rectum & 115 & $33(29.7)$ & $40(36.0)$ & $42(37.8)$ & \\
\hline Pathological type & & & & & 0.093 \\
\hline Adenocarcinoma & 323 & $105(94.6)$ & $109(98.2)$ & $110(99.1)$ & \\
\hline Others & 9 & $6(5.4)$ & $2(1.8)$ & I (0.9) & \\
\hline Chemotherapy regimen & & & & & 0.457 \\
\hline Oxaliplatin-based & 244 & $84(75.7)$ & $80(72.1)$ & $80(72.1)$ & \\
\hline Irinotecan-based & 80 & $25(22.5)$ & $26(23.4)$ & $29(26.1)$ & \\
\hline Fluorouracil alone & 8 & I (0.9) & $5(4.5)$ & $2(1.8)$ & \\
\hline Other type & 1 & I (0.9) & & & \\
\hline Bevacizumab addition or not & & & & & 0.375 \\
\hline Yes & 87 & $24(21.6)$ & $33(29.7)$ & $30(27.0)$ & \\
\hline No & 246 & 87 (78.4) & $78(70.3)$ & $81(73.0)$ & \\
\hline
\end{tabular}

Abbreviation: $\mathrm{LDH}$, lactate dehydrogenase.

aggravate the hypoxia (through pruning the tumor vessels) but also, facilitate aerobic glycolysis via increase in the expression of the key enzyme, pyruvate kinase M2. ${ }^{10,35,36}$ Meanwhile, LDH is also strongly related to both the hypoxia environment and aerobic glycolysis. The $L D H$ gene, among hundreds of genes activated by the $H I F$ gene, could be upregulated under hypoxic conditions. ${ }^{22}$ Lactate, the main product of aerobic glycolysis, is closely associated with the change of LDH as well. ${ }^{37}$ Therefore, we explored the potential value of LDH to predict the efficacy of bevacizumab. Recently, LDH has shown promising value for predicting the efficacy of bevacizumab and its analog, vatalanib.
However, the two drugs are not identical, and the evidence regarding LDH cannot be shared. This hypothesis comes from the relationship between tyrosine kinase inhibitors (TKIs) and monoclonal antibodies in non-small cell lung cancer (NSCLC) treatments. TKIs for epidermal growth factor receptor (EGFR), including gefitinib, erlotinib, and ecotinib, have been shown to be effective for NSCLC patients bearing mutant EGFR, and the mutant EGFR is a powerful predictor of TKI efficacy;38 however, cetuximab, the monoclonal antibody for EGFR, is not so effective and lack of predictors in consensus, though all of them target EGFR receptor. ${ }^{39}$ As for bevacizumab, only
A

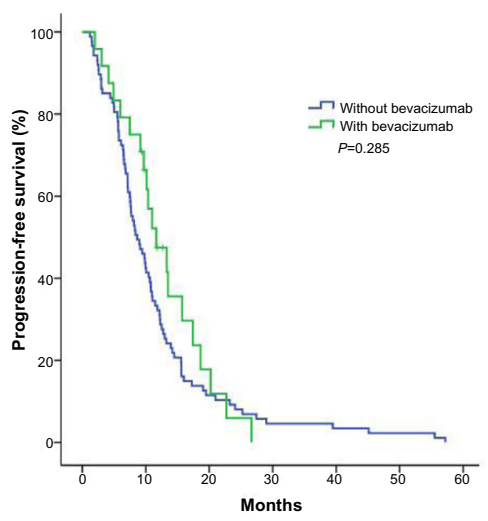

B

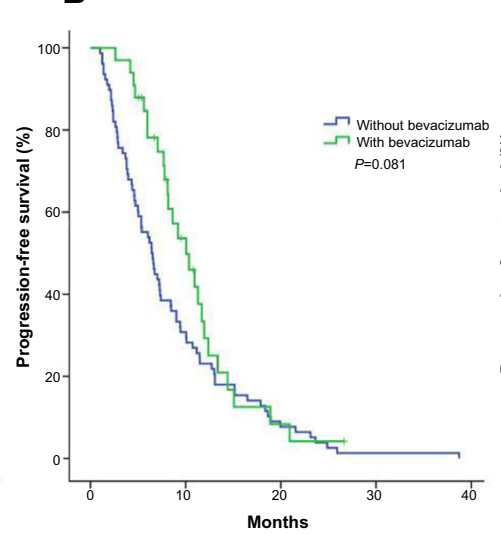

C

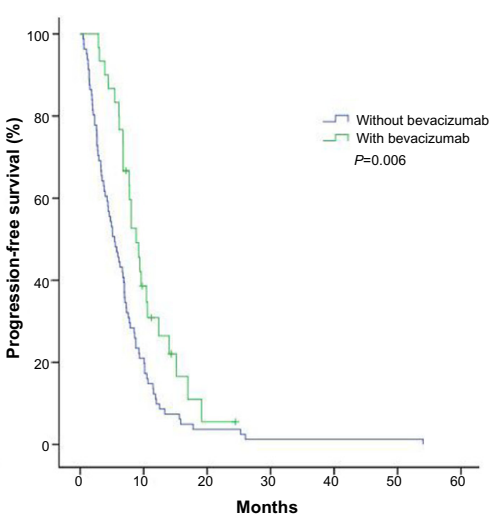

Figure 6 (A) The PFS of the one-third of patients, with and without bevacizumab, in the low serum LDH group; (B) the PFS of the one-third of patients, with and without bevacizumab, in the middle serum LDH group; (C) the PFS of the one-third of patients, with and without bevacizumab, in the high serum LDH group. Abbreviations: LDH, lactate dehydrogenase; PFS, progression-free survival. 


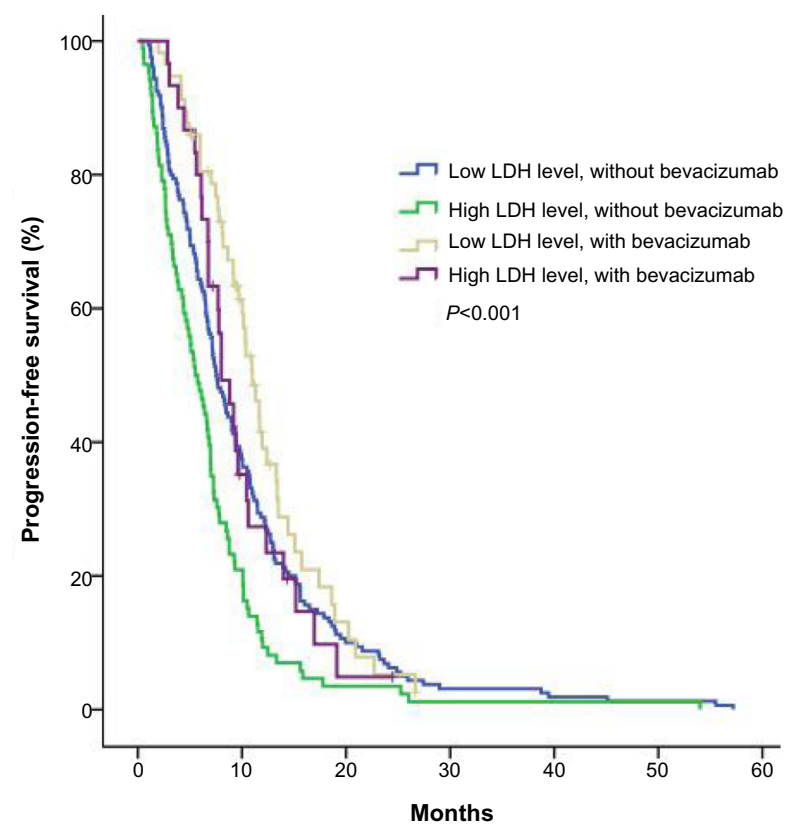

Figure 7 The PFS of the patients, with and without bevacizumab, in the low serum $\mathrm{LDH}$ and high level groups.

Abbreviations: LDH, lactate dehydrogenase; PFS, progression-free survival.

one paper addressed whether LDH could predict its efficacy in mCRC. However, there are several differences between it and our study ${ }^{23}$ First, in our study, patients who received less than four cycles of bevacizumab were excluded since their short exposure may have lowered the potential for survival benefit. Second, in the previous work, the cutoff value for pretreatment LDH was determined using a receiver operating characteristics curve analysis based on the selected patients, and this may have biased the result in that the LDH cutoff value may not have represented the overall group of mCRC patients, ${ }^{23}$ in our study, the cutoff value for LDH was based on routine clinical examination, not only obtained by large scale clinical research but also, practical. Our study demonstrated that different levels of serum LDH could distinguish the PFS of the patients who received bevacizumab from those who did not, which is similar to the previous study. ${ }^{23}$ More importantly, our study found that in the high LDH level group, patients who received bevacizumab had statistically significant improvement of PFS, while in the LDH low group, it brought no benefit. This finding may hint that LDH level should be tested before deciding whether bevacizumab could be administrated for $\mathrm{mCRC}$ patients, although this should be validated in prospective study. This finding was validated in our further investigation that classified patients into three groups according to LDH level, where the difference between receipt of bevacizumab or not changed from insignificant to significant according to the increase of LDH level.
In conclusion, the addition of bevacizumab to standard first-line chemotherapy can bring PFS benefit in Chinese mCRC patients; however, the benefit may be only limited to patients with initial high LDH level. Meanwhile, LDH level was found to be a poor prognostic factor for mCRC. However, all the findings in the current study should be validated in studies with larger sample size, and the OS should be calculated if the follow-up time permits.

\section{Acknowledgments}

This article was supported by grants from Science and Technology Planning Project of Guangdong Province, People's Republic of China (2011B061300069) and National Natural Science Foundation of China (81272641 and 81071872).

\section{Author contributions}

All authors contributed toward data analysis, drafting and revising the paper and agree to be accountable for all aspects of the work.

\section{Disclosure}

The authors report no conflicts of interest in this work.

\section{References}

1. Hurwitz H, Fehrenbacher L, Novotny W, et al. Bevacizumab plus irinotecan, fluorouracil, and leucovorin for metastatic colorectal cancer. $N$ Engl J Med. 2004;350(23):2335-2342.

2. Saltz LB, Clarke S, Díaz-Rubio E, et al. Bevacizumab in combination with oxaliplatin-based chemotherapy as first-line therapy in metastatic colorectal cancer: a randomized phase III study. J Clin Oncol. 2008;26(12):2013-2019.

3. Giantonio BJ, Catalano PJ, Meropol NJ, et al; Eastern Cooperative Oncology Group Study E3200. Bevacizumab in combination with oxaliplatin, fluorouracil, and leucovorin (FOLFOX4) for previously treated metastatic colorectal cancer: results from the Eastern Cooperative Oncology Group Study E3200. J Clin Oncol. 2007;25(12):1539-1544.

4. Bennouna J, Sastre J, Arnold D, et al; ML18147 Study Investigators. Continuation of bevacizumab after first progression in metastatic colorectal cancer (ML18147): a randomised phase 3 trial. Lancet Oncol. 2013;14(1):29-37.

5. Guan ZZ, Xu JM, Luo RC, et al. Efficacy and safety of bevacizumab plus chemotherapy in Chinese patients with metastatic colorectal cancer: a randomized phase III ARTIST trial. Chin J Cancer. 2011;30(10): 682-689.

6. Rugo HS. Inhibiting angiogenesis in breast cancer: the beginning of the end or the end of the beginning? J Clin Oncol. 2012;30(9):898-901.

7. Miles DW, Chan A, Dirix LY, et al. Phase III study of bevacizumab plus docetaxel compared with placebo plus docetaxel for the first-line treatment of human epidermal growth factor receptor 2-negative metastatic breast cancer. J Clin Oncol. 2010;28(20):3239-3247.

8. Robert NJ, Diéras V, Glaspy J, et al. RIBBON-1: randomized, doubleblind, placebo-controlled, phase III trial of chemotherapy with or without bevacizumab for first-line treatment of human epidermal growth factor receptor 2-negative, locally recurrent or metastatic breast cancer. J Clin Oncol. 2011;29(10):1252-1260.

9. Sasich LD, Sukkari SR. The US FDAs withdrawal of the breast cancer indication for Avastin (bevacizumab). Saudi Pharm J. 2012;20(4):381-385 
10. Jain RK. Normalizing tumor vasculature with anti-angiogenic therapy: a new paradigm for combination therapy. Nat Med. 2001;7(9): 987-989.

11. Gatenby RA, Gillies RJ. Why do cancers have high aerobic glycolysis? Nat Rev Cancer. 2004;4(11):891-899.

12. Busk M, Horsman MR. Relevance of hypoxia in radiation oncology: pathophysiology, tumor biology and implications for treatment. Q J Nucl Med Mol Imaging. 2013;57(3):219-234.

13. Anastasiou D, Poulogiannis G, Asara JM, et al. Inhibition of pyruvate kinase M2 by reactive oxygen species contributes to cellular antioxidant responses. Science. 2011;334(6060):1278-1283.

14. Kostakis ID, Vaiopoulos AG, Philippou A, Papavassiliou AG, Koutsilieris M, Kouraklis G. Preoperative serum lactate dehydrogenase levels in colorectal and gastric cancer: a hospital-based case-control study. Biomark Med. 2013;7(1):131-137.

15. Kayser G, Kassem A, Sienel W, et al. Lactate-dehydrogenase 5 is overexpressed in non-small cell lung cancer and correlates with the expression of the transketolase-like protein 1. Diagn Pathol. 2010;5:22.

16. Agarwala SS, Keilholz U, Gilles E, et al. LDH correlation with survival in advanced melanoma from two large, randomised trials (Oblimersen GM301 and EORTC 18951). Eur J Cancer. 2009;45(10):1807-1814.

17. Wang ZY, Loo TY, Shen JG, et al. LDH-A silencing suppresses breast cancer tumorigenicity through induction of oxidative stress mediated mitochondrial pathway apoptosis. Breast Cancer Res Treat. 2012;131(3):791-800.

18. Danner BC, Didilis VN, Wiemeyer S, et al. Long-term survival is linked to serum LDH and partly to tumour LDH-5 in NSCLC. Anticancer Res. 2010;30(4):1347-1351.

19. Hong J, Yoon HH, Ahn HK, et al. Prognostic role of serum lactate dehydrogenase beyond initial diagnosis: a retrospective analysis of patients with diffuse large B cell lymphoma. Acta Haematol. 2013;130(4):305-311.

20. Armstrong AJ, George DJ, Halabi S. Serum lactate dehydrogenase predicts for overall survival benefit in patients with metastatic renal cell carcinoma treated with inhibition of mammalian target of rapamycin. $J$ Clin Oncol. 2012;30(27):3402-3407.

21. Abaza H, Ghanem A, Jmal A, et al. [Importance of determination of $\mathrm{C}$ reactive protein (CRP), carcinoembryonic antigen (CEA) and lactic dehydrogenase (LDH) in colorectal cancer]. Tunis Med. 2010;88(6):409-413. French.

22. Koukourakis MI, Giatromanolaki A, Sivridis E, et al. Prognostic and predictive role of lactate dehydrogenase 5 expression in colorectal cancer patients treated with PTK787/ZK 222584 (vatalanib) antiangiogenic therapy. Clin Cancer Res. 2011;17(14):4892-4900.

23. Scartozzi M, Giampieri R, Maccaroni E, et al. Pre-treatment lactate dehydrogenase levels as predictor of efficacy of first-line bevacizumabbased therapy in metastatic colorectal cancer patients. Br J Cancer. 2012;106(5):799-804.

24. Hecht JR, Trarbach T, Hainsworth JD, et al. Randomized, placebocontrolled, phase III study of first-line oxaliplatin-based chemotherapy plus PTK787/ZK 222584, an oral vascular endothelial growth factor receptor inhibitor, in patients with metastatic colorectal adenocarcinoma. J Clin Oncol. 2011;29(15):1997-2003.
25. Van Cutsem E, Bajetta E, Valle J, et al. Randomized, placebo-controlled, phase III study of oxaliplatin, fluorouracil, and leucovorin with or without PTK787/ZK 222584 in patients with previously treated metastatic colorectal adenocarcinoma. J Clin Oncol. 2011;29(15):2004-2010.

26. Zhang B, He W, Zhou F, Guo G, Jiang C, et al. A Potential Administration-time Dependent Effect of Bevacizumab in Improving Overall Survival and Increasing Metastasis in Metastatic Colorectal Cancer. Chemotherapy. 2013;2:108.

27. Giantonio BJ, Catalano PJ, Meropol NJ, et al. Eastern Cooperative Oncology Group Study E3200. Bevacizumab in combination with oxaliplatin, fluorouracil, and leucovorin (FOLFOX4) for previously treated metastatic colorectal cancer: results from the Eastern Cooperative Oncology Group Study E3200. J Clin Oncol. 2007;25(12):1539-1544.

28. Li G, Gao J, Tao YL, et al. Increased pretreatment levels of serum LDH and ALP as poor prognostic factors for nasopharyngeal carcinoma. Chin J Cancer. 2012;31(4):197-206.

29. Koukourakis MI, Giatromanolaki A, Sivridis E, et al. Lactate Dehydrogenase 5 Expression in Operable Colorectal Cancer: Strong Association With survival and Activated Vascular Endothelial Growth Factor Pathway-A Report of the Tumour Angiogenesis Research Group. J Clin Oncol. 2006;24:4301-4308.

30. Fahmueller YN, Nagel D, Hoffmann RT, Tatsch K, Jakobs T, Stieber $\mathrm{P}$, Holdenrieder S. Predictive and prognostic value of circulating nucleosomes and serum biomarkers in patients with metastasized colorectal cancer undergoing Selective Internal Radiation Therapy. $B M C$ Cancer. 2012;12:5.

31. Zhang B, He W, Zhou F, et al. A potential administration-time dependent effect of Bevacizumab in improving overall survival and increasing metastasis in metastatic colorectal cancer. 2013;2(1):1-5.

32. Wu Q, Shi Y, Chen L, Xiao X, Dai G. Effect and safety of bevacizumabcontaining chemotherapy treatment in Chinese patients with metastatic colorectal cancer. Onco Targets Ther. 2013;6:485-490.

33. Kim Y, Stolarska MA, Othmer HG. The role of the microenvironment in tumor growth and invasion. Prog Biophys Mol Biol. 2011;106(2): 353-379.

34. Folkman J. Tumor angiogenesis: role in regulation of tumor growth. Symp Soc Dev Biol. 1974;30(0):43-52.

35. Christofk HR, Vander Heiden MG, Wu N, Asara JM, Cantley LC. Pyruvate kinase M2 is a phosphotyrosine-binding protein. Nature. 2008;452(7184):181-186

36. Vander Heiden MG, Cantley LC, Thompson CB. Understanding the Warburg effect: the metabolic requirements of cell proliferation. Science. 2009;324(5930):1029-1033.

37. Feron O. Pyruvate into lactate and back: from the Warburg effect to symbiotic energy fuel exchange in cancer cells. Radiother Oncol. 2009;92(3):329-333.

38. Mok TS, Wu YL, Thongprasert S, et al. Gefitinib or CarboplatinPaclitaxel in Pulmonary Adenocarcinoma. N Engl J Med. 2009;361:947957

39. Dassonville O, Bozec A, Fischel JL, Milano G. EGFR targeting therapies: monoclonal antibodies versus tyrosine kinase inhibitors. Similarities and differences. Crit Rev Oncol Hematol. 2007;62(1):53-61.
OncoTargets and Therapy

\section{Publish your work in this journal}

OncoTargets and Therapy is an international, peer-reviewed, open access journal focusing on the pathological basis of all cancers, potential targets for therapy and treatment protocols employed to improve the management of cancer patients. The journal also focuses on the impact of management programs and new therapeutic agents and protocols on
Dovepress

patient perspectives such as quality of life, adherence and satisfaction The manuscript management system is completely online and includes a very quick and fair peer-review system, which is all easy to use. Visit http://www.dovepress.com/testimonials.php to read real quotes from published authors. 